\title{
PLATFORM SESSION 2: IMAGING AND SENTENCE COMPREHENSION
}

\author{
Syntactic and Semantic Processes in the Intact Brain: \\ An Event-Related Study with fMRI
}

\author{
Weijia Ni,*,† Donald Shankweiler, $*,+\mathrm{R}$. Todd Constable, $\dagger$ \\ W. Einar Mencl, $* \dagger$ Kenneth R. Pugh, $* \dagger$ Robert K. Fulbright, $\dagger$ \\ Sally E. Shaywitz, $\dagger$ Bennett A. Shaywitz, $\dagger$ and John C. Gore $\dagger$ \\ *Haskins Laboratories, †Yale University School of Medicine, \\ and $¥$ University of Connecticut
}

Since the 1970s, much research effort has been expended in attempts to distinguish contributions of anterior and posterior parts of the Sylvian region and its surround to specific processes in sentence production and comprehension. Neurolinguistic studies have sought to demonstrate that the anterior part of this zone (including but not necessarily limited to Broca's area) is a focus for syntactic abilities (e.g., Caramazza \& Zurif, 1976) and the posterior part (including but not limited to Wernicke's area) represents semantic and lexical abilities. Although there is some support for this synthesis of the clinical findings, there is a concern that data from damaged brains yield ambiguous answers to questions regarding functional organization within the language cortex.

Techniques that record brain activity while neurologically intact subjects are engaged in language tasks offer a way to avoid some of these ambiguities. Monitoring the electrical activity of the brain by recording event-related potentials (ERP) yields possible indicators of (different aspects of) sentence processing. P600 has been proposed as an indicator of syntactic parsing because it is triggered by violations of grammaticality; similarly, N400 has been hypothesized as an indicator of semantic interpretation because it is triggered at the point where a sentence stops making sense (Hagoort, Brown, \& Osterhout, 1999). Consensus has not been reached regarding these and other ERP indicators, however. The range of phenomena that elicit them is still unclear. Moreover, localization of ERP waveforms is difficult.

Functional magnetic neuroimaging (fMRI) offers the needed spatial resolution. Recently, fMRI has been adapted to make it capable of detecting infrequent events, expanding the potential of brain-imaging studies to exploit 
the kinds of sentence-processing tasks that are standard in psycholinguistic research. Results of two experiments using the same stimulus materials are presented: One follows the conventional method of blocking stimulus materials of the same type; the other exploits the newer event-related methodology.

While in the scanner, 14 young, unimpaired adults listened to simple sentences that were either nonanomalous or contained a syntactic anomaly (e.g., *Trees can grew.) or a semantic anomaly (e.g., *Trees can cry.).

Anomaly detection. Subjects judged sentences for acceptability. Same/ different pitch judgments provided a baseline. The purpose was to separate linguistic processing from other aspects of the task (attention, auditory processing, and memory). fMRI signals evoked by baseline pitch judgments were subtracted from signals generated during separate blocks of sentence trials, each containing syntactic or semantic anomalies. The residual activity isolates linguistic processes. Activation was bilateral, more in the left hemisphere than in the right, in regions surrounding the Sylvian fissure, including the inferior frontal area anteriorly and the middle and superior temporal area posteriorly. These comprise a major portion of the language zone identified by studies of aphasic persons and electrical stimulation of the cortex (Ojemann, 1983). There is much overlap between regions activated by each type of anomaly.

Event-related single-trial paradigm. Subjects heard isolated occurrences of anomalous sentences interspersed among nonanomalous sentences. Judgments of animacy were made, which did not require anomaly detection. The aims were to determine whether effects of isolated occurrences of anomalous sentences are detectable and whether syntactic and semantic anomalies can be differentiated when attention is directed elsewhere. Regional activation associated with each type of sentence anomaly was clearly differentiated. Syntactic anomalies activated the inferior frontal region strongly and posterior regions minimally. Semantic anomalies showed a complementary pattern, activating posterior temporal areas strongly and the inferior frontal area weakly.

The results of both experiments agree with other evidence in finding sites surrounding the Sylvian region specifically active during sentence processing. The clear separation of linguistic activity from nonlinguistic activity associated with the experimental tasks comports well with the hypothesis of a brain specialization for language, a central claim of the modularity view of language (Fodor, 1983; Liberman \& Mattingly, 1989). Further, syntactic operations are most strongly associated with sites in the inferior frontal gyrus, and semantic operations are most strongly associated with sites in the posterior temporal gyrus (see Fig. 7). This dissociation is consistent with evidence from ERP (Hagoort et al., 1998) and online psycholinguistic studies (Ni et al., 1998) that syntactic computations are automatic, encapsulated, and separate from semantic interpretation.

The critical region for syntax is a part of the premotor cortex. Rizzolatti 


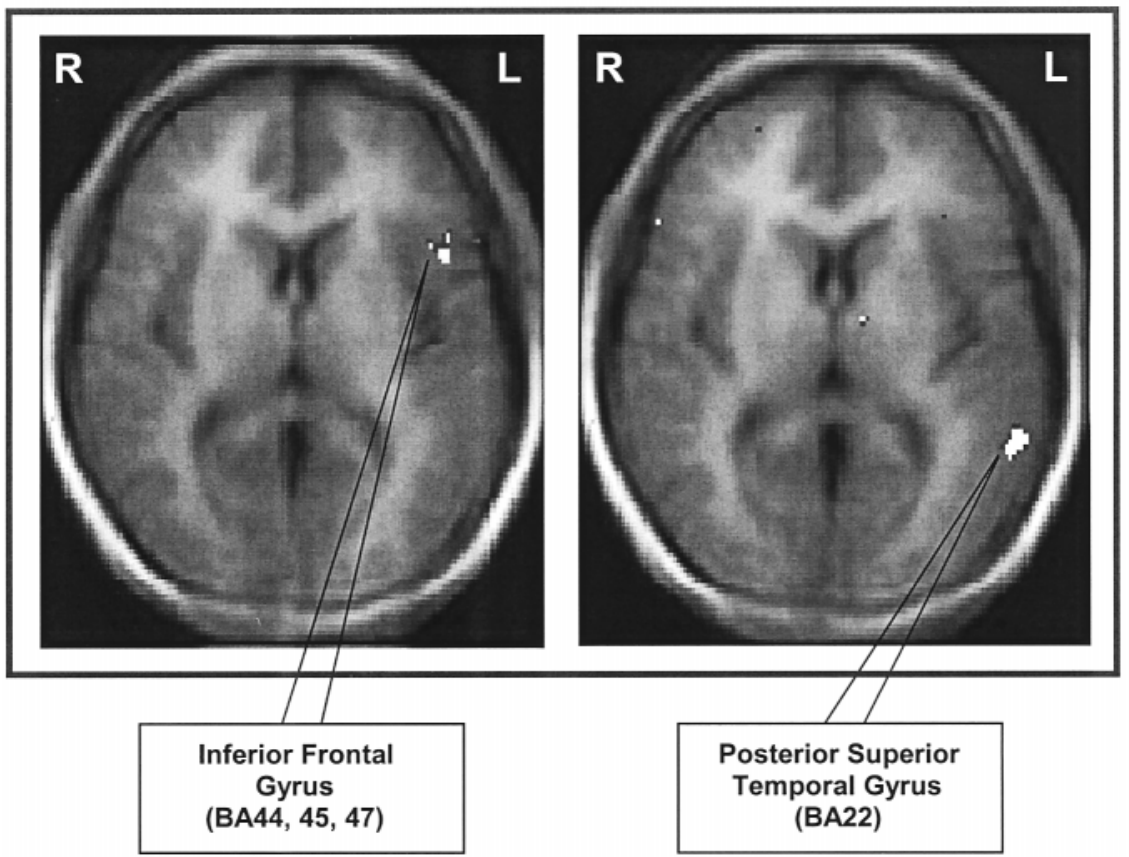

FIG. 7. Dissociation of brain activity revealed by the event-related single-trial paradigm. Sites (averaged over 14 subjects) that were specifically activated as listeners experienced syntactic (left) and semantic (right) anomalies while they were attending to an irrelevant aspect of the sentences (animacy).

and Arbib (1998) propose that this inferior frontal region is the analog of the region in the monkey brain where "mirror neurons," cells that fire when the monkey is either executing or observing communicative gestures, are located. The hypothetical link with language, and specifically syntax, is through imitation of sequences of motor gestures, a principle of organization of the language modality and a possible mechanism for language evolution (Studdert-Kennedy, 1999).

\section{REFERENCES}

Caramazza, A., \& Zurif, E. 1976. Dissociation of algorithmic and heuristic processes in language comprehension: Evidence from aphasia. Brain and Language, 3, 572-582.

Fodor, J. A. 1983. Modularity of mind. Cambridge, MA: MIT Press.

Hagoort, P., Brown, C., \& Osterhout, L. 1998. The neurocognition of syntactic processing. In C. Brown and P. Hagoort (Eds.), Neurocognition of language. London: Oxford Univ. Press.

Liberman, A. M., \& Mattingly, I. G. 1989. A specialization for speech perception. Science, 243, 489-494. 
Ni, W., Fodor, J. D., Crain, S., \& Shankweiler, D. 1998. Anomaly detection: Eye-movement patterns. Journal of Psycholinguistic Research, 27, 515-539.

Ojemann, G. A. 1983. Brain organization for language from the perspective of electrical stimulation mapping. Behavioral and Brain Sciences, 2, 189-230.

Rizzolatti, G., \& Arbib, M. 1998. Language within our grasp. Trends in Neurosciences, 21, $188-194$.

Studdert-Kennedy, M. 1999. Evolutionary implications of the particulate principle: Imitation and the dissociation of phonetic form from semantic function. In C. Knight, M. StuddertKennedy, \& J. R. Hurford (Eds.), The emergence of language: Social function and the origins of linguistic form. Cambridge, UK: Cambridge Univ. Press, in press.

\section{The Role of the Left Anterior Temporal Cortex in Language Processing}

\section{Peter Hagoort,* Nick Ramsey, $\dagger$ Geert-Jan Rutten, $\dagger$ and Peter van Rijen $\dagger$}

*Max Planck Institute for Psycholinguistics, Nijmegen; and †University Hospital of Utrecht, Utrecht, The Netherlands

Introduction. Recent studies suggest a role for the anterior left temporal cortex in sentence-level comprehension. In a PET study, Mazoyer et al. (1993) found left and right temporal pole activation in three different sentence conditions, but not in word lists. They ascribe these activations to syntactic parsing or prosodic analysis. Dronkers et al. (1994, 1990; see also Hagoort et al., 1999) found $100 \%$ lesion overlap in the left anterior superior temporal gyrus in nine patients with poor morphosyntactic comprehension. More specifically, the lesioned area was the anterior portion of Brodmann area 22. In the Dronkers et al. study morphosyntactic comprehension was mainly tested in a series of sentence-picture matching tasks.

Both studies suggest a role for the left anterior temporal cortex in sentencelevel processing, but do not allow firm conclusions about the precise aspects of sentence-level processing that the left anterior temporal cortex might subserve. In addition, classically this area has not been part of the network of core areas for language production and comprehension.

This study aimed at further investigating the role of the left anterior temporal cortex in language processing. The approach was, however, different. We tested five patients in whom the left anterior temporal lobe was removed to cure an intractable epilepsy on a battery of language tests, including sentence-level comprehension and, more specifically, syntactic comprehension.

Subjects. Five right-handed patients (four males) participated in this study. All patients were operated because of an intractable temporal lobe epilepsy which had lasted for 25 years on average (range 18-36). All patients had left mesial temporal sclerosis with no other structural lesions. The mean age of the patients at time of operation was 32 years (range 19-47). Before the 
operation none of the patients showed any sign of a language deficit. All patients had left hemisphere dominance for language as determined by a preoperative bilateral intracarotid amytal (Wada) test. To localize languagecritical areas with subcentimeter precision during operation, intraoperative electrocortical stimulation mapping was performed while the patient performed a picture-naming task (Debets et al., 1991; Ojemann et al., 1993). No significant naming errors were found in the cortical areas that were later resected. The resection included the left amygdala-hippocampus complex and the anterior part of the left temporal neocortex. Starting from the temporal pole, the resection size of the superior temporal gyrus and the middle temporal gyrus was between 4 and $5 \mathrm{~cm}$; the resection size of the inferior temporal gyrus was between 4 and $6 \mathrm{~cm}$.

Methods. Testing of the patients was done not earlier than 22 months after operation and not later than 50 months after operation. The patients were administered the Dutch version of the Aachen Aphasia Test (AAT). The AAT contains subtests for single word and sentence understanding in the auditory and visual modality, subtests for word and sentence repetition, naming of objects and situations, writing, the Token Test, and a standardized way of collecting a sample of spontaneous speech.

In addition to the AAT, a specific test for syntactic comprehension was done. This test was a Dutch adaptation of a German test of syntactic comprehension (Huber et al., 1993). In this syntactic task subjects had to select one of four target pictures on the basis of an auditorily presented sentence. The syntactic test consisted of five types of sentences in Dutch (a total of 72 items), which differed in their degree of syntactic complexity. The first type consisted of active, semantically irreversible sentences ("The girl with the ribbon carries the ball.' ). The second type consisted of active, semantically reversible sentences ("The man with the ball is looking for the child."). The third type were simple passive sentences ("The man with the ball is being looked for by the child."'). The fourth type were sentences with an active subject relative clause ("The child that is looking for the man has a ball."). The most complex sentence type consisted of sentences with a passive subject relative clause ("The child that is being looked for by the man has a ball.' ). If the subject explicitly asked for a repetition, the sentence was read again. Responses were scored on a 3-point scale: 2 points for correctly matched sentences, 1 point for sentences that were correctly matched after self-correction and for sentences that were presented twice, and 0 points for incorrectly matched sentences. The maximum score was 144 .

Results. The spontaneous speech of the patients was normal. Mean length of utterance, well-formedness of sentences, sentence prosody, etc., were all normal for the five patients. The results of the Token Test were for all patients within the normal range. Four patients made no errors, one patient made 1 error (of 50 possible). Repetition of sounds, words, and sentences was also normal in all five patients. Three patients scored within the normal range 
on the comprehension test of the AAT (with scores of 112, 119, and 115, respectively; maximum score 120). Two patients showed a minor language comprehension problem, with scores of 104 and 99. These two patients (SS and DV) were, however, not worse on sentence comprehension compared to single word comprehension. Naming of objects and situations was normal in four patients. One patient (DV) had a minor naming problem, especially for compound nouns and for naming situations.

The syntactic offline test resulted for all five patients in scores that were within the normal range. The average score was 132 (92\% correct), with a range between $128(89 \%)$ and $139(97 \%)$. A group of 12 normal controls that we tested on the same syntactic offline test had an average score of 137 (95\%). Even on the most complex syntactic structures, the performance of the patients was within the normal range.

Discussion. None of the patients in this study showed any evidence for an impairment at the level of written and spoken sentence-level comprehension. The minor remaining problems in two patients in naming and comprehension are most likely due to memory problems that resulted from the operation. All patients were fully normal in using syntactic information for sentence interpretation. These data, therefore, do not support earlier claims that the left anterior temporal cortex contributes to parsing or other sentencelevel processes such as prosodic analysis. The data support the classical model in which the left anterior temporal cortex is not assumed to be critical for core language functions. The only caveat is that one does not know for sure whether the brains of these patients are fully representative of the normal organization of language in the brain, as the resected temporal cortex partly consisted of epileptogenic tissue. Therefore, some reorganization might have taken place. Nevertheless, clearly more and stronger evidence is needed than has been presented so far, to ascribe a role in language processing to the temporal pole and anterior portions of the superior temporal gyrus.

\section{REFERENCES}

Debets, R. M., van Veelen, C. W. M., van Huffelen, A. C., \& van Emde Boas, W. 1991. Presurgical evaluation of patients with intractable partial epilepsy: The Dutch epilepsy surgery program. Acta Neurologica Belgica, 91, 125-140.

Dronkers, N. F., Wilkins, D. P., Van Valin, R. D., Jr., Redfern, B. B., \& Jaeger, J. J. 1994. A reconsideration of the brain areas involved in the disruption of morphosyntactic comprehension. Brain and Language, 46, 461-463.

Dronkers, N. F., Wilkins, D. P., Van Valin, R. D., Jr., Redfern, B. B., \& Jaeger, J. J. 1999. Cortical areas underlying the comprehension of grammar. Submitted for publication.

Hagoort, P., Brown, C. M., \& Osterhout, L. 1999. The neurocognition of syntactic processing. In C. M. Brown \& P. Hagoort (Eds.), The neurocognition of language (pp. 273-318). London: Oxford Univ. Press.

Huber, W., Klingenberg, G., Poeck, K., \& Willmes, K. 1993. Die Supplemente zum Aachener Aphasie Test-Aufbau and Resultate der Validierung. Neurolinguistik, 7, 43-66. 
Mazoyer, B. M., Tzourio, N., Frak, V., Syrota, A., Muruyama, N., Levrier, O., Salamon, G., Dehaene, S., Cohen, L., \& Mehler, J. 1993. The cortical representation of speech. Journal of Cognitive Neuroscience, 5, 467-479.

Ojemann, G. A., Sutherling, W. W., Lesser, R. P., Dinner, D. S., Jayakar, P., \& Saint-Hillaire, J.-M. 1993. Cortical stimulation. In J. Engel Jr. (Ed.), Surgical treatment of the epilepsies (pp. 399-414). New York: Raven Press.

\title{
Sentence Listening with and without Responding
}

\author{
Steven L. Small,* Martha W. Burton,* Charles A. Perfetti, $\dagger$ \\ and Douglas C. Noll\$
}

*Department of Neurology, University of Maryland; †Department of Psychology, University of Pittsburgh; and $\$$ Department of Biomedical Engineering,

University of Michigan

Introduction. The present study investigates the functional neuroanatomy of auditory sentence processing using fMRI. We address the presence of distributed processing networks by studying three different types of "sentences" that might be expected to involve different brain processing mechanisms. Specifically, by examining the different neuroanatomical substrates underlying the processing of grammatical and scrambled sentences, we expect to uncover those regions involved in the syntactic and semantic processing of sentences. The contrast between auditorily presented sentences and similar nonlinguistic auditory stimuli (i.e., digital reversals of the same sentences) aims to determine the extent to which activation patterns can be attributed to language processing per se or to more general aspects of processing continuous speech-like stimuli. By comparing these three neural networks, we aim to characterize the auditory language comprehension system in terms of prosodic processing, word sequence processing, and sentence processing.

Although there are now several studies that have asked subjects to listen to sentences without responding immediately, the pattern of brain activation with passive sentence listening remains unclear (Binder, Rao, Hammeke, Yetkin, Jesmanowicz, Bandettini, et al., 1994; Kawashima, Itoh, Hatazawa, Miyazawa, Yamada, Matsuzawa, et al., 1993; Mazoyer, Tzourio, Frak, Syrota, Murayama, Levrier, et al., 1993; Muller, Rothermel, Behen, Muzik, Mangner, \& Chugani, 1997; Schlosser, Aoyagi, Fulbright, Gore, \& McCarthy, 1998).

The research described here used whole-brain fMRI during two sentence listening tasks in normal subjects to uncover the different components and the lateralization of the distributed neural system involved in auditory sentence comprehension. We compared listening passively (Experiment 1) to listening for specific information (i.e., monitoring sentences for semantic 
content) (Experiment 2). Both experiments included three types of auditory stimuli (grammatical sentences, scrambled sentences, and digitally reversed sentences) and rest.

The "rest" condition was aimed at providing a baseline control to aid in data interpretation by surveying the overall pattern of distributed brain activation during stimulus presentation, prior to making any comparisons according to the controversial "subtraction" method (Burton \& Small, 1999).

Experiment 1: Passive listening. Nine normal right-handed native English speakers (mean age 31 years) participated in the study. The grammatical and scrambled sentence stimuli (Miller \& Isard, 1963) were five, six, seven, and nine words long and included 50 meaningful grammatical sentences and an equal number of scrambled sentences (constructed by reshuffling the grammatical sentences) and reverse sentences (constructed by digitally reversing the grammatical sentences).

Stimulus presentation was blocked, with subjects asked to perform one of the three different auditory "sentence" processing tasks for $20 \mathrm{~s}$, followed by rest, followed by a different active task, with the entire trial consisting of 18 active blocks.

Functional image acquisition and analysis were performed according to previously described methods, including a group average image constructed by applying a Fisher transformation to each stereotactically transformed correlation image and then averaging together the (Gaussian) smoothed result (see Burton \& Small, 1999, for a review of these methods).

All subjects recalled specific sentences from the experiment, indicating that they actively participated in the task. The imaging results are shown in Fig. 8. There was no significant activation comparing sentence types to each other. Compared to rest, passive listening to grammatical, scrambled, or reverse sentences was associated with a single active left hemisphere region, centered in the superior temporal gyrus (STG) very close to the primary auditory cortex. In the right hemisphere, two temporal areas, the STG and the anterior superior temporal sulcus (STS), were active on all stimulus types. Listening to grammatical or scrambled stimuli also activated the posterior STS. Scrambled and reverse sentences also activated a region in the posterior STG.

Experiment 2: Sentence monitoring forced response. The similarity of the reverse speech condition to the two sentence conditions suggests that subjects might have been attending to the auditory characteristics of the stimuli, but not to the sentential syntax and semantic aspects of the stimuli. By adding monitoring tasks to the same sentence stimuli, Experiment 2 addressed whether the processing of the three types of sentences would reveal a distinct neural substrate when attentional and memory demands were increased.

In Experiment 2, the same three stimulus types were presented, with an active motor response (i.e., button press) required. For sentences, this was 
Left
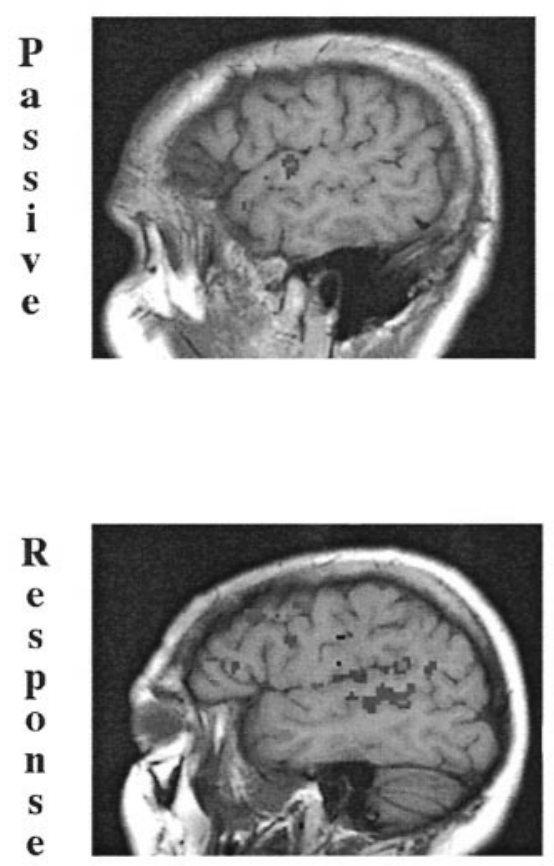

Right
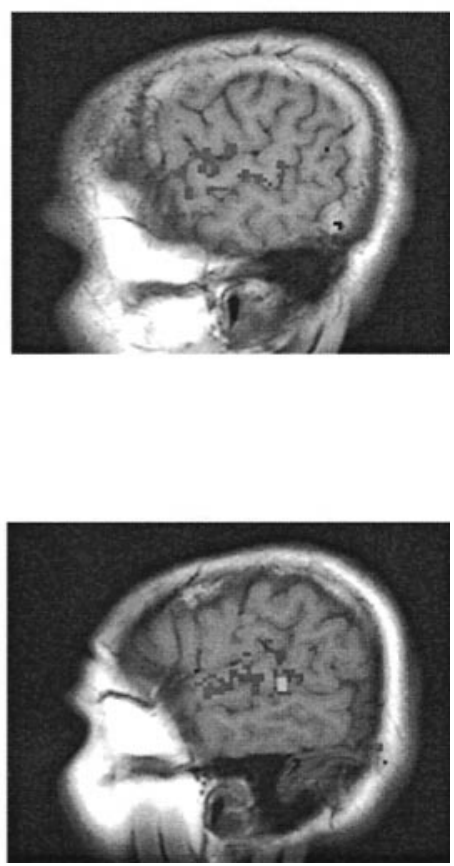

FIG. 8. Brain activation pattern on passive listening and listening with forced response to grammatical sentences in the one subject. Both tasks show bilateral distributed temporal activation. Note that additional activation in the left angular and middle frontal regions is present with the increased memory and semantic processing demands of the forced response task.

a semantic category judgment ("transportation"). For scrambled and reverse sentences, this required tone monitoring.

Nine normal right-handed native English speakers (mean age 33 years) participated in the study. All methods were the same as those used in Experiment 1, except for the response requirement.

Subjects achieved greater than $90 \%$ accuracy, with 2 of the 15 sentences leading to virtually all errors. Response times showed grammatical sentences to be significantly faster than reverse or scrambled sentences. Data analysis occurred as in Experiment 1.

The comparisons of grammatical, scrambled, and reverse sentences with rest all demonstrated similar bilateral anatomical networks of activity, with predominance of the STG and STS in both hemispheres (as in passive listening). Grammatical and scrambled sentences activated the left angular gyrus (AG), and grammatical sentences activated the left middle frontal gyrus (MFG). 
Compared to monitoring digitally reversed speech for a tone, semantic monitoring of grammatical sentences activated three brain regions, the left posterior STS, the left MFG, and the superior aspect of the right cerebellar hemisphere. The comparison of scrambled and reverse sentence monitoring activated a small area of the left STS within the larger area found in the comparison of grammatical and reverse sentence monitoring.

Discussion. The two experiments described here demonstrate that sentence comprehension processes recruit a broad network of perisylvian cortical regions in both cerebral hemispheres, particularly in the STG and STS. When sentence listening is accompanied by a task requiring semantic interpretation, this broad bilateral network is augmented by left lateralized activation in the STS, the MFG, and the AG. It is not known whether this added activity relates to aspects of syntactic or semantic processing or working memory.

\title{
REFERENCES
}

Binder, J. R., Rao, S. M., Hammeke, T. A., Yetkin, F. Z., Jesmanowicz, A., Bandettini, P. A., Wong, E. C., Estkowski, L. D., Goldstein, M. D., Haughton, V. M., \& Hyde, J. S. 1994. Functional magnetic resonance imaging of human auditory cortex. Annals of Neurology, 35, 662-672.

Burton, M. W., \& Small, S. L. 1999. An introduction to fMRI. Neurologist, in press.

Kawashima, R., Itoh, M., Hatazawa, J., Miyazawa, H., Yamada, K., Matsuzawa, T., \& Fukuda, H. 1993. Changes of regional cerebral blood flow during listening to an unfamiliar spoken language. Neuroscience Letters, 161(1), 69-72.

Mazoyer, B. M., Tzourio, N., Frak, V., Syrota, A., Murayama, N., Levrier, O., Salamon, G., Dehaene, S., Cohen, L., \& Mehler, J. 1993. The cortical representation of speech. Journal of Cognitive Neuroscience, 5(4), 467-479.

Miller, G. A., \& Isard, S. 1963. Some perceptual consequences of linguistic rules. Journal of Verbal Learning and Verbal Behavior, 2, 217-228.

Muller, R. A., Rothermel, R. D., Behen, M. E., Muzik, O., Mangner, T. J., \& Chugani, H. T. 1997. Receptive and expressive language activations for sentences: A PET study. NeuroReport, 8(17), 3767-3770.

Schlosser, M. J., Aoyagi, N., Fulbright, R. K., Gore, J. C., \& McCarthy, G. 1998. Functional MRI studies of auditory comprehension. Human Brain Mapping, 6(1), 1-13.

\section{The Role of Broca's Area and the Frontal Operculum in Language Comprehension}

\author{
Angela D. Friederici, Martin Meyer, and D. Yves von Cramon
}

Max Planck Institute of Cognitive Neuroscience

There is a long-standing discussion of the role of Broca's area in language production and comprehension. It has been argued that it is not damage to the lateral Broca's area as such that causes persistent Broca's aphasia, but 
that the entire fronto-opercular cortex needs to be damaged to produce Broca's aphasia (Mohr et al., 1978) and/or that the anterior insula needs to be involved (Dronkers et al., 1994). These claims are based on data from traditional lesion-behavior studies. More recently imaging techniques allow one to investigate which particular language functions are subserved by these distinct cortical areas in the healthy brain.

The present study used event-related functional magnetic resonance imaging at $3 \mathrm{~T}$ to specify the functions supported by distinct brain areas. The stimulus material was constructed to allow the investigation of semantic, syntactic, and phonological processes. It comprised four different conditions: (1) normal sentences, (2) jabberwocky sentences containing syntactic structure but no semantic meaning (content words were replaced by pseudowords), (3) lists of real words containing semantic meaning but no structural information, and (4) lists of phonologically legal pseudowords containing neither semantic nor syntactic information.

Subjects' task was to listen carefully to each trial and to perform two judgments, (a) whether it contained syntactic structure and (b) whether it was meaningful. Performance on these judgments was very high, indicating that subjects understood the stimuli well.

During listening to the different stimulus types the hemodynamic response elicited by each trial was obtained from eight horizontal slices parallel to the AC-PC line covering the entire perisylvian cortex by means of a multisclice echo planar imaging sequence.

Eighteen healthy university students (age range 20-26 years) participated in the experiment.

Different activation patterns were analyzed for the different experimental conditions allowing the specification of a cerebral network subserving auditory language processing. All auditory input types caused activation in Heschl's gyri, in the planum temporale bilaterally, and in the lateral segment of the superior temporal gyrus. The data suggest that phonological processing, in addition, is correlated with an increased activation in the cortex lining the junction area of the inferior precentral sulcus and the inferior frontal sulcus in the left hemisphere. The right hemisphere homologue corresponding to this area was active for all but the pseudoword condition, suggesting an involvement of this area (among others) in semantic processes. Specific brain activation was observed for the two syntactic conditions compared to the word list conditions in the posterior portion of the temporal operculum (planum polare) bilaterally. The frontal operculum (not the crown region of the Broca's area) was particularly engaged in the jabberwocky condition containing only syntactic information.

From these data we conclude that the neuronal network supporting syntactic processing includes parts of temporal as well as left frontal cortices and that the frontal operculum, in particular, plays a critical role in the processing of syntactic structure. 


\section{REFERENCES}

Dronkers, N., Wilkins, D. P., Van Vilin, R. D., Redfern, B. B., \& Jaeger, J. J. 1994. A reconsideration of the brain areas involved in the disruption of morphosyntactic comprehension. Brain and Language, 47, 461-463.

Mohr, J. P., Pessin, M. S., Finkelstein, S., Funkenstein, H. H., Duncan, G. W., \& Davis, M. D. 1978. Broca aphasia: Pathologic and clinical. Neurology, 28, 311-324.

\section{Online Syntactic Processing in Aphasia: Studies with Auditory Moving Window Presentation}

\section{David Caplan* and Gloria Waters $\dagger$}

*Neuropsychology, Massachusetts General Hospital; and †Communication Disorders, Boston University

Introduction. Aphasic patients often have impairments in comprehending semantically reversible sentences with complex syntactic structures, such as passives and object-relativized forms (Caplan et al., 1985). These disorders are thought to result from abnormalities affecting specific types of syntactic representations and/or associated parsing operations and from reductions in available "processing resources" that cause syntactic computations to crash prematurely (see Caplan and Hildebrandt, 1988 for overview). These models of syntactic comprehension deficits thus attribute the deficit to disorders that affect online construction of one or more aspect of a syntactic representation and/or the use of such representations to determine aspects of propositional content. Some data, however, suggest that some aphasic patients process syntactic structures normally online and that their syntactic comprehension impairments arise at a later stage of processing (Tyler, 1985). The present study investigates online processing of syntactic form using a new methodauditory moving window.

Subjects. Twenty aphasic subjects and an equal number of age- and education-matched normal controls were tested. Aphasic patients all had left hemisphere vascular lesions and were at least 6 months postevent. Their aphasic symptoms were evaluated using standard clinical assessment tools, and they were classified into clinically recognized aphasic types.

Method. Online sentence processing efficiency was assessed using the Auditory Moving Window (AMW) paradigm. Subjects heard a spoken sentence that had been digitized and segmented into a series of phrases. Their task was to pace their way through the sentence as quickly as possible, by pressing a button on a box interfaced with the computer for the successive presentation of each phrase, and then to make an acceptability judgment about the sentence they had just heard. Reaction times for each button press, as well as response time and accuracy on the acceptability judgment, were recorded. 
The target stimuli consisted of 104 semantically acceptable and 104 semantically unacceptable sentences divided equally among cleft-subject (CS; It was/ the food/ that nourished/ the child), cleft-object (CO; It was/ the child/ that the food/ nourished), object-subject (OS; The father/ read/ the book/ that terrified/ the child), and subject-object (SO; The book/ that the father/ $\mathrm{read} /$ terrified/ the child) sentences. CO and SO are more complex than CS and OS sentences, respectively, and the locus of the complexity has been shown to be the embedded verb of the relative clause (V1) and the second (main) verb in the SO (V2) (Gibson, 1998). Thus, the main data of interest were listening times for the verb in acceptable CO compared to CS sentences and for V1 and V2 in acceptable SO compared to OS sentences.

Results. Aphasic patients made more errors and took longer on the judgment task than controls, as shown in Fig. 9, top. For both patients and controls, there were more errors and responses were longer for $\mathrm{CO}$ and $\mathrm{SO}$ than for CS and OS sentences, respectively.

Listening times (minus segment durations) are shown in Fig. 9, bottom. The expected increase in listening times at the verb of CO compared to CS sentences and at both verbs of SO compared to OS sentences was found. There was also an increase in listening times at the last word of SO compared to OS sentences. As a group, there was no difference in listening times between aphasic and control subjects.

Aphasic patients were divided into high- and low-comprehension groups on the basis of their accuracy performances. Figure 9, top, shows that the low-comprehension aphasic group made more errors and took longer on the judgment task. Figure 9, bottom, shows that, with one exception, these two groups performed virtually identically on the online listening portion of the AMW task, with both showing the expected increases in listening time on the more complex segments of the more complex sentences. The exception is that the high-comprehension group showed the normal increase in reading times on V1 of SO compared to OS sentences, while the low-comprehension group did not.

Aphasic patients were also divided into fluent and nonfluent groups. There were no differences in the performances of these groups of patients.

Discussion. The auditory moving windows task has been shown to be sensitive to factors that make for local sentence complexity, such as lexical frequency (Ferreira et al., 1996a), syntactic ambiguity (Ferreira et al., 1996b), and syntactic structural complexity (Caplan and Waters, 1999). The results of this study continue to show that listening times are sensitive to local syntactic complexity, since they increase at points in sentences at which additional computations are required. The results of this study indicate that aphasic patients show almost the same increases in listening times as control subjects on these more demanding portions of the sentence, despite not understanding the sentences as well as controls.

What do these data suggest about aphasic impairments? One possibility 
Number of Errors
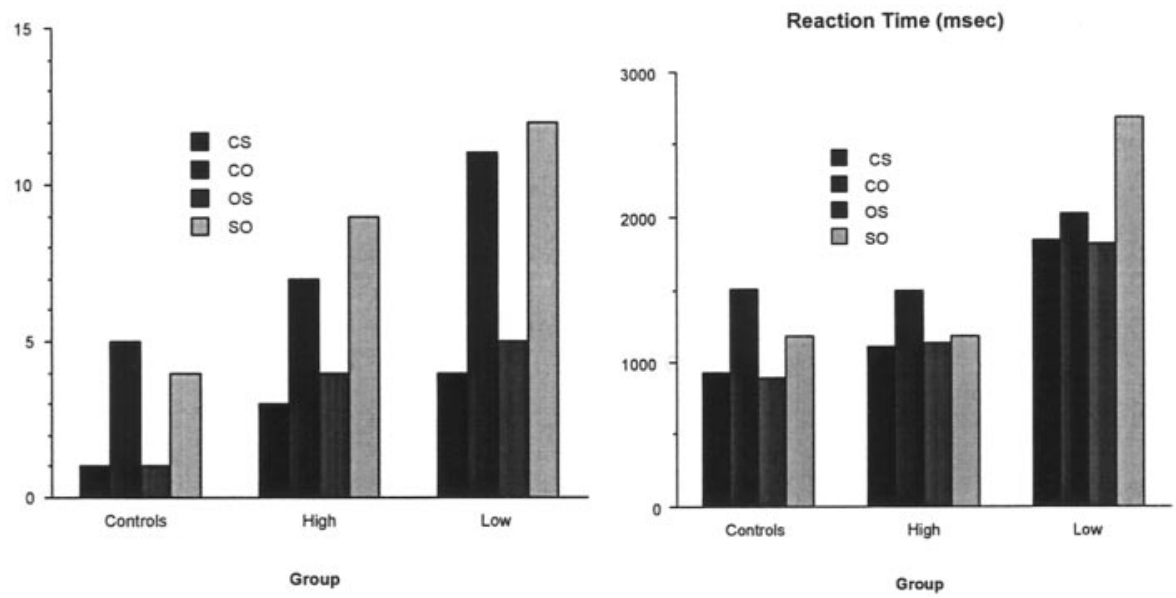

Cleft Object - Cleft Subject
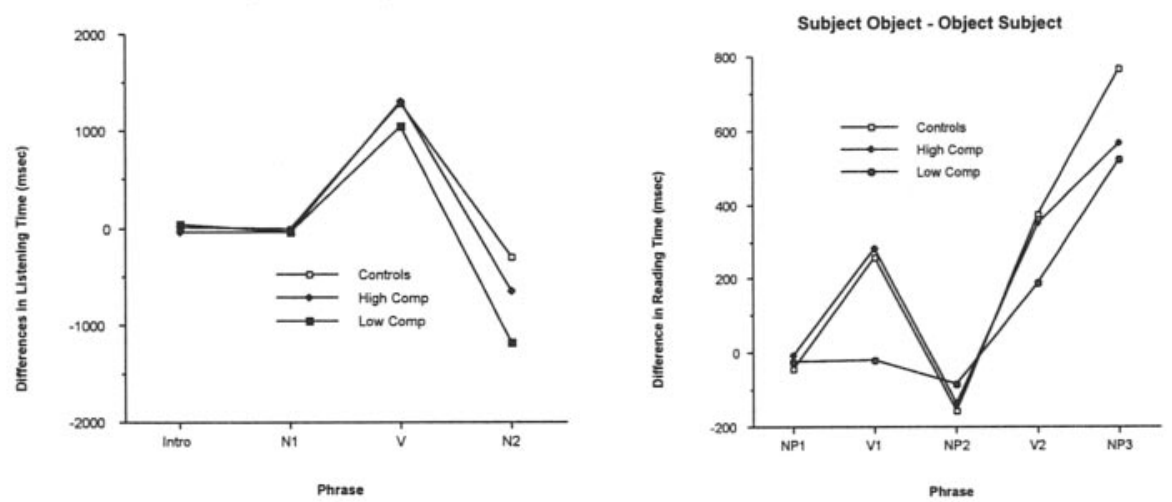

FIGURE 9

is that aphasic patients create abnormal, simpler structures on the basis of incoming segments of sentences and that this process takes exactly as long (relative to a simpler sentence baseline) as the process of creating more complex, normal structures on the part of normal subjects. Another possibility is that these patients fail to structure some segments of sentences and that they cease trying to structure these segments at exactly the same time as normal subjects create a normal structure. These possibilities strike us as overly reliant on coincidence, but either may be correct. A third possibility is that many of these patients create normal syntactic and sentential semantic structures, and their impairments largely reflect offline cognitive or metacognitive impairments. If so, since these impairments are worse for syntactically more complex sentences, the form of a sentence, as well as its meaning, 
must influence these cognitive functions. Our results raise the possibility that this is the case.

\section{REFERENCES}

Caplan, D., \& Hildebrandt, H. 1988. Disorders of syntactic comprehension. Cambridge, MA: MIT Press.

Caplan, D., \& Waters, G. S. 1999. Verbal working memory and sentence comprehension, BBS, in press.

Caplan, D., et al. 1985. Cognition, 21, 117-175.

Ferreira, F., et al. 1996. Journal of Psycholinguistic Research, 25, 273-290.

Ferreira, F., et al. 1996b. Journal of Experimental Psychology: Learning, Memory, \& Cognition, 22, 324-335.

Gibson, E. 1998. Cognition, 68, 1-76.

Tyler, L. 1985. Brain \& Language, 26, 259-275. 
